\title{
Quark Propagators in the confinement and the deconfinement phases
}

\section{Masatoshi Hamada*, Masanobu Yahiro}

Kyushu University, Fukuoka, Japan

\section{Hiroaki Kouno}

Saga University, Saga, Japan

\section{Atsushi Nakamura}

Hiroshima University, Hiroshima, Japan

\section{Takuya Saito}

Kochi University, Kochi, Japan

E-mail: tsaito@rcnp.osaka-u.ac.jp

\begin{abstract}
We calculated quark propagators in the confinement and the deconfinement phases by quenched lattice QCD simulation with the clover improved fermion. The effective masses from the vector part are estimated on several lattice sizes. The masses obtained in units of $T_{c}$ show a rapid change after the QGP phase transition.
\end{abstract}

The XXVI International Symposium on Lattice Field Theory

July 14-19 2008

Williamsburg, Virginia, USA

\footnotetext{
* Speaker.
} 


\section{Introduction}

It is important to study quark propagators not only in the confinement phase but also the deconfinement phase. The quark confinement means that the isolated quark spectrum absents in the asymptotic state. The information of the asymptotic state appears in the propagators corresponding particles. It is expected that quark propagators behave as something unpredicted by perturbative QCD in the confinement phase. In the deconfinement phase, it is expected that the quark has the quasi-particle state as the asymptotic state. The mass of the quasi-particle is called thermal mass defined at the chiral limit $\left(m_{q} \rightarrow 0\right)$ and is calculated in the high temperature limit.

There are several lattice calculations of quark propagators $[2,3,4,5,6,7,8]$. In the confinement phase, the motivation to study quark propagators is whether infrared singularities exist or not in the momentum space. These calculations were summarized by Ref. [2]. On the other hand, studies in the deconfinement phase are not so much. However, the existence of plasmino-mode near $T_{c}$ is suggested in the lattice calculation[8].

We here carry out the $S U(3)$ lattice simulation with the clover improved Wilson fermion to investigate the quark propagator near the QCD phase transition point. The thermalized quenched lattice configurations are fixed by the Landau gauge. In this report we plot the effective mass of the quark propagator in the QGP medium and discuss the qualitative difference between the confinement and deconfinement phases.

\section{Formulation}

We assume that a quark propagator is defined by a simple one pole form,

$$
G\left(p_{4}\right)=\frac{Z\left(p_{4}\right)}{i \not p_{4}+m}=\frac{Z\left(p_{4}\right)\left(-i \not p_{4}+m\right)}{p_{4}^{2}+m^{2}},
$$

where $m$ is the pole mass, $Z$ is the renormalization function and $p_{4}$ is the time-like momentum. The propagator in the co-ordinate space is defined by Fourier transformation,

$$
G(t) \equiv\langle\psi(t) \bar{\psi}(0)\rangle=\int \frac{d p_{4}}{2 \pi} G\left(p_{4}\right) e^{i p_{4} t},
$$

where the time-like momentum $p_{4}$ 's are equal to $\left(2 n_{t}-1\right) \pi / N_{t}$ with $n_{t}=1,2, \cdots N_{t}$ for the antiperiodic boundary condition in temporal direction. The quark propagator in the co-ordinate space has the following form,

$$
\begin{array}{r}
G(t)=\frac{Z}{2 \cosh (m / 2 T)}\left[\cosh (m(t-1 / 2 T)) \gamma_{4}\right. \\
-\sinh (m(t-1 / 2 T))],
\end{array}
$$

where $1 / T=N_{t}$ and $t$ is the temporal extension.

It is important information that in the co-ordinate space quark propagators with some pole structure behave as the sum of propagators with one pole. In addition, the form of quark propagators at $\vec{p}=0$ is written by

$$
G(t)=G_{4}(t) \gamma_{4}+G_{s}(t) .
$$


Table 1: Parameters

\begin{tabular}{cccc|cccc}
\hline$\beta$ & temperature & $\kappa$ & $C_{S W}$ & $\beta$ & temperature & $\kappa$ & $C_{S W}$ \\
\hline 6.10 & 1.08998 & 0.1345559 & 1.6787 & 6.25 & 1.36650 & 0.1346226 & 1.5876 \\
& & 0.1353591 & 1.6787 & & & 0.1352633 & 1.5876 \\
\hline
\end{tabular}
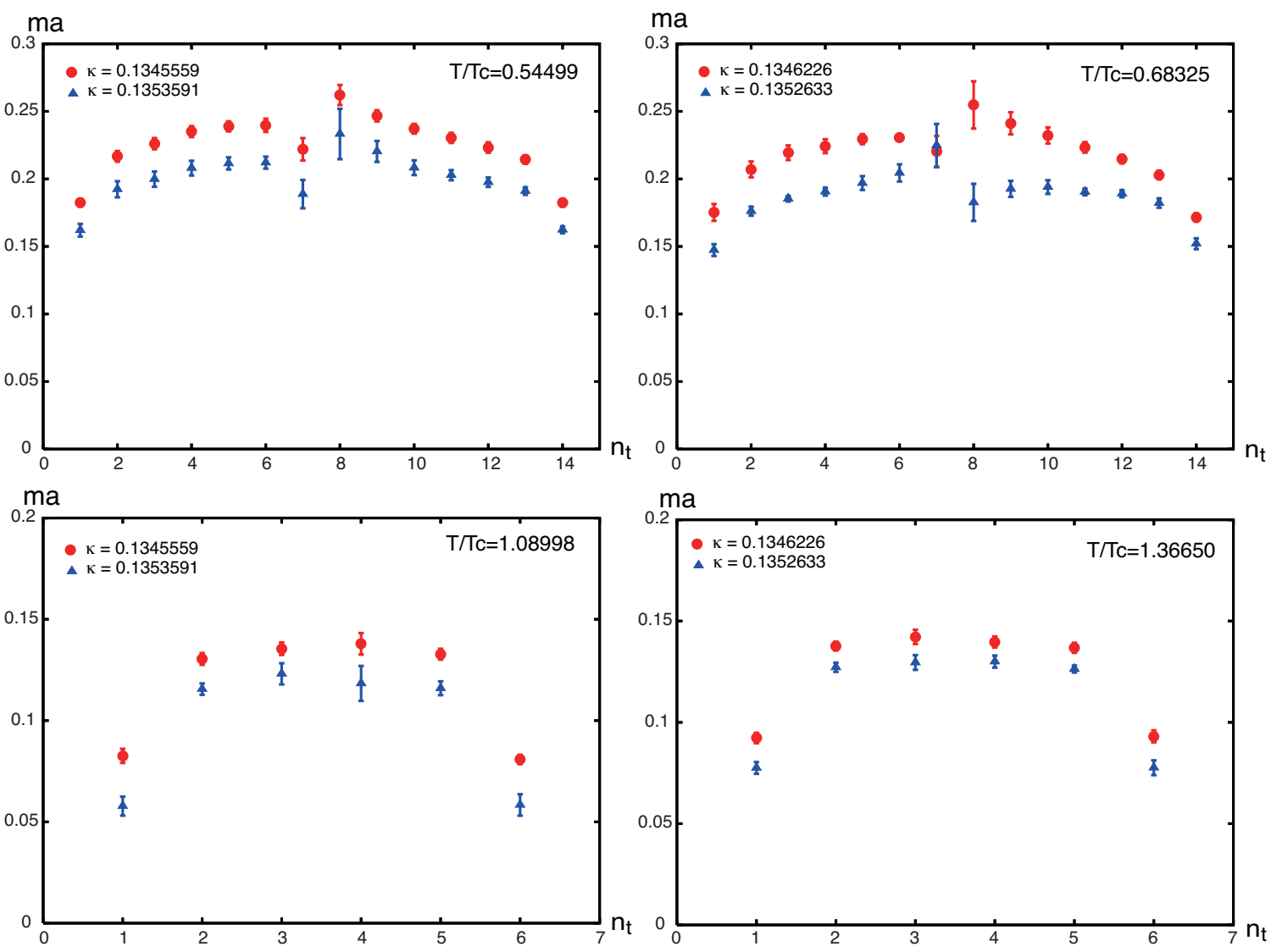

Figure 1: Effective masses defined by the vector part of quark propagators in the confinement and deconfinement phases. In those figures, two top figures correspond to results in the confinement phase $\left(N_{s}=24\right.$ and $\left.N_{t}=16\right)$. Two bottom figures correspond to results in the deconfinement phase $\left(N_{s}=24\right.$ and $\left.N_{t}=8\right)$. $\beta=6.10$ for the left figures and $\beta=6.25$ for the right figures.

The effective mass is defined by the ratios,

$$
G_{4}(t) / G_{4}(t+1), \quad G_{s}(t) / G_{s}(t+1) .
$$

If propagators have only one pole, the effective mass $m_{\text {eff }}$ obtained by Eq. (2.5) should be a constant as $t$ increases.

\section{Numerical results}

Figure 1 shows the behavior of the effective masses defined by the vector part of quark propa- 

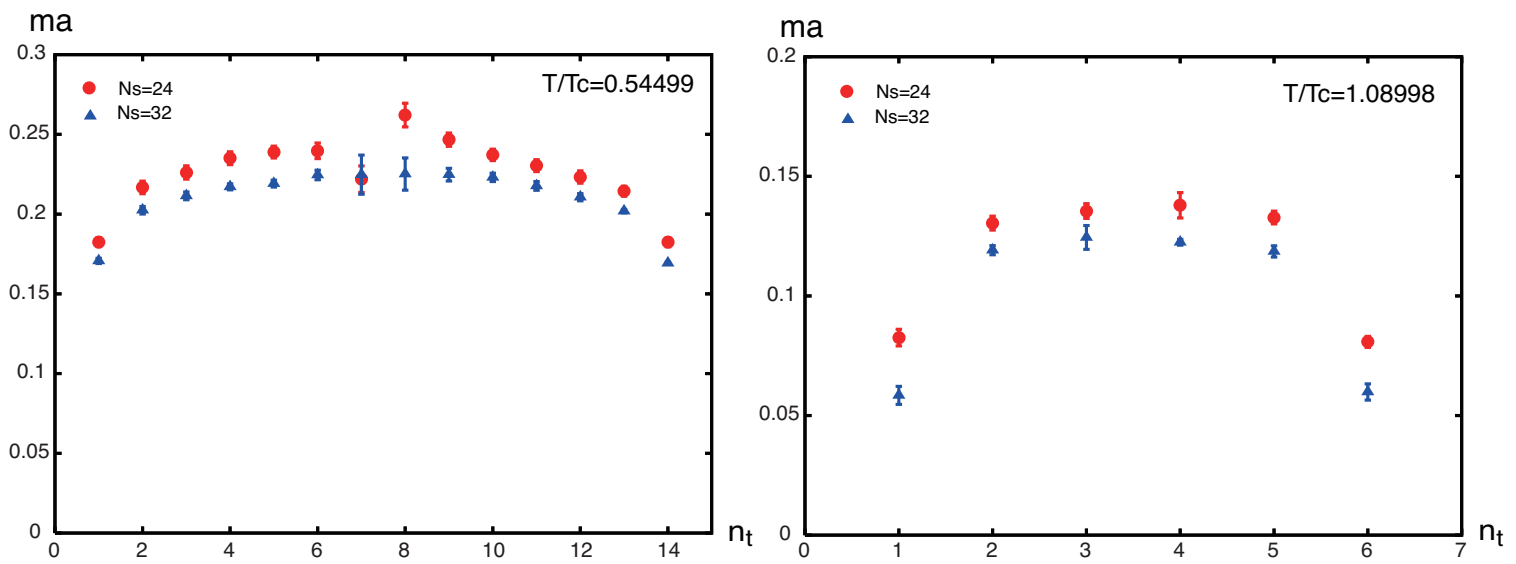

Figure 2: They show the volume dependence of the effective mass defined by the vector part of quark propagators. In those figures, $N_{s}=24$ and $N_{s}=32, \beta=6.10$ and $\kappa=0.1345559$. The left figure corresponds to the result of the confinement phase $\left(N_{t}=16\right)$. The right figure corresponds to the result of the deconfinement phase $\left(N_{t}=8\right)$. The effective mass decreases as volume increases in both the confinement and the deconfinement phases.

gators in the confinement (top two figures) and the deconfinement (bottom two figures) phases. In those calculations, the temporal lattice size $\left(N_{t}\right)$ is equal to 16 in the confinement phase and 8 in the deconfinement phase and the spatial lattice size $\left(N_{s}\right)$ is equal to 24 .

The effective mass in the confinement phase approaches a flat from the under as time increases. This behavior is different from that of normal particles like mesons. In the case of mesons or some other particles, the effective mass approaches a flat from the over as time increase. We can understand what is the reason of this strange behavior if quark propagators have pole structure. The effective mass is roughly written by

$$
m_{e f f}=m_{0}+\ln \left(\frac{1+\rho \exp (-\Delta m t)}{1+\rho \exp (-\Delta m(t+1))}\right),
$$

where $m_{0}$ is the mass of the lowest mode, $\Delta m$ is the difference between the mass of the lowest and the next modes and $\rho$ is the ratio of the norm of the lowest and the next modes. The behavior such as two top figures in Fig. 1 is realized with $\rho$ is negative; that is, quark propagators contain negative norm states. The effective mass in the deconfinement phase is constant excepting $n_{t}=1$ and $n_{t}=6$. The behavior suggests that negative norm states absent in the deconfinement phase.

Figure 3 shows the temperature dependence of the effective mass, which is roughly constant in both the confinement and the deconfinement phases respectively and the mass in the confinement phase is larger than one in the deconfinement phase.

Up to this, we only show the effective mass defined by the vector part of quark propagators. The scalar part is ill-fitted in the case of $N_{t} \leq 16$. Figure 4 calculated by the parameters $\beta=6.00$, $\kappa=0.1335080, N_{s} \times N_{t}=24^{3} \times 24,36^{3} \times 36$ suggests that the scalar part is well-fitted in large $N_{t}$ calculations. The right figure in Fig. 4 shows that the both masses defined by the vector part and the scalar part are approximately the same value at large $n_{t}$. It is a reasonable result because the vector part and the scalar part of quark propagators in the form of Eq. (2.4) have the same information about the pole mass. 


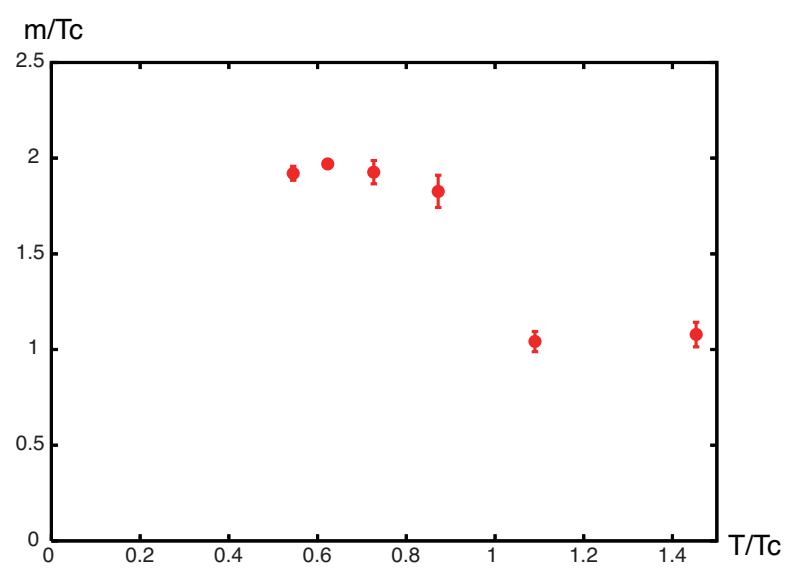

Figure 3: This shows the temperature dependence of the effective mass. In this figure, $N_{s}=32, N_{t}=$ $16,14,12,10,8,6, \beta=6.10$, and $\kappa=0.1345559$. Results of $N_{t}=16,14,12,10$ correspond to the mass below $T_{c}$ and results of $N_{t}=8,6$ correspond to the mass above $T_{c}$. Those masses are defined by flat parts of the effective masses.
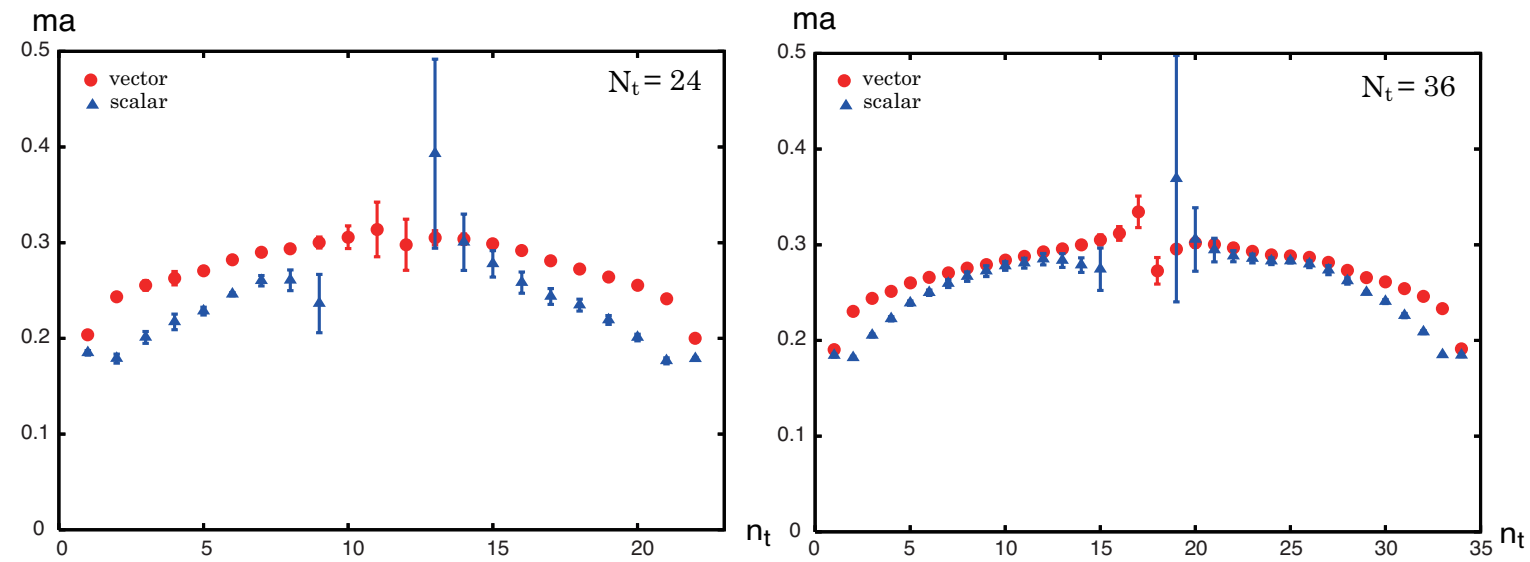

Figure 4: They show the lattice size dependence of the effective mass with $\beta=6.00$ and $\kappa=0.1335080$. In those cases we can fit not only the vector part of quark propagators but also the scalar part. The right figure shows that the effective mass defined by the scalar part approaches to one defined by the vector part.

\section{Summary}

We studied quark propagators in the confinement and the deconfinement phases. The temporal lattice size needs to be sufficiently large (at least $N_{s} \geq 24$ to fit well) to analyze the scalar part of quark propagators. Here we fixed the gauge with Landau gauge condition. However, it is known that the Landau gauge condition violates the positivity of the transfer matrix but it might be maintained only in the Coulomb gauge condition that the effective mass defined by the exponential damp of quark propagators is the pole mass[10]. Therefore we should calculate quark propagators with the Coulomb gauge condition as the next step. 


\section{Acknowledgments}

We thank F. Karsch and M. Kitazawa for useful comments. The simulation has been done on the SX8 at Research Center for Nuclear Physics, Osaka University, the SR11000 at Hiroshima University, RIKEN Super Combined Cluster (RSCC) at Riken and the kyu-cc at Kyushu University.

\section{References}

[1] M. Le Bellac, Thermal Field Theory(Cambrigde University Press, Cambridge, 2000)

[2] P.O. Bowman, U.M. Heller, D.B. Leinweber, A.G. Williams, and J.B. Zhang, Lattice Hadron Physics, Lecture Notes (Springer-Verlag).

[3] W. Kamleh, P.O. Bowman, D.B. Leinweber, A.G. Williams, J. Zhang, arXiv:hep-lat/0705.4129.

[4] S. Furui, arXiv:hep-lat/0801.0325

[5] G. Boyd, F. Karsch, S. Gupta, Nucl.Phys.B385, 481, (1992).

[6] P. Petreczky, F. Karsch, E. Laermann, S. Stickan, I.Wetzorke, Nucl.Phys.Proc.Suppl. 106, 513 (2002).

[7] M Hamada, H Kouno, A Nakamura, T Saito, M Yahiro, PoS(LATTICE 2006)136.

[8] F. Karsch, M. Kitazawa, Phys. Lett. B658, 45, (2007) and PoS(LATTICE 2007)197.

[9] B. Sheikholeslami and R. Wohlert, Nucl. Phys. B259, 572 (1985).

[10] O. Philipsen, Phys.Lett. B521, 273, (2001). 